\title{
ON FINITENESS PROPERTIES ON ASSOCIATED PRIMES OF LOCAL COHOMOLOGY MODULES AND EXT-MODULES
}

\author{
Lizhong Chu AND Xian Wang
}

ABstRACT. Let $R$ be a commutative Noetherian (not necessarily local)
ring, $I$ an ideal of $R$ and $M$ a finitely generated $R$-module. In this
paper, by computing the local cohomology modules and Ext-modules
via the injective resolution of $M$, we proved that, if for an integer $t>0$,
$\operatorname{dim}_{R} H_{I}^{i}(M) \leq k$ for $\forall i<t$, then
\[ \bigcup_{i=0}^{j}\left(\operatorname{Ass}_{R} H_{I}^{i}(M)\right)_{\geq k}=\bigcup_{i=0}^{j}\left(\operatorname{Ass}_{R} \operatorname{Ext}_{R}^{i}\left(R / I^{n}, M\right)\right)_{\geq k} \] for $\forall j \leq t$ and $\forall n>0$. This shows that $\bigcup_{n>0}\left(\operatorname{Ass}_{R} \operatorname{Ext}_{R}^{i}\left(R / I^{n}, M\right)\right)_{\geq k}$ is a finite set for $\forall i \leq t$. Also, we prove that

$\bigcup_{i=1}^{r}\left(\operatorname{Ass}_{R} M /\left(x_{1}^{n_{1}}, x_{2}^{n_{2}}, \ldots, x_{i}^{n_{i}}\right) M\right)_{\geq k}=\bigcup_{i=1}^{r}\left(\operatorname{Ass}_{R} M /\left(x_{1}, x_{2}, \ldots, x_{i}\right) M\right)_{\geq k}$

if $x_{1}, x_{2}, \ldots, x_{r}$ is $M$-sequences in dimension $>k$ and $n_{1}, n_{2}, \ldots, n_{r}$ are some positive integers. Here, for a subset $T$ of $\operatorname{Spec}(R)$, set $T_{\geq i}=\{\mathfrak{p} \in$ $T \mid \operatorname{dim} R / \mathfrak{p} \geq i\}$

\section{Introduction}

Throughout this paper, let $R$ be a commutative Noetherian ring and $I$ an ideal of $R$. Let $M$ be a finitely generated $R$-module. For convenience, we use the following notations: for a subset $T$ of $\operatorname{Spec}(R)$, we set

$$
\begin{aligned}
T_{\geq i} & :=\{\mathfrak{p} \in T \mid \operatorname{dim} R / \mathfrak{p} \geq i\}, \\
T_{>i} & :=\{\mathfrak{p} \in T \mid \operatorname{dim} R / \mathfrak{p}>i\}, \\
T_{i} & :=\{\mathfrak{p} \in T \mid \operatorname{dim} R / \mathfrak{p}=i\} .
\end{aligned}
$$

It is well-known that the sequences of associated primes $\operatorname{Ass}_{R} M / I^{n} M$ and $\operatorname{Ass}_{R} I^{n} M / I^{n+1} M, n=1,2, \ldots$ eventually become constant for $n>>0$ (see

Received January 16, 2013; Revised August 27, 2013.

2010 Mathematics Subject Classification. 13D45, 13C15.

Key words and phrases. local cohomology modules, associated primes, $M$-sequences in dimension $>k$.

This work is supported by Natural Science Foundation of China (11201326), by the Natural Science Foundation of Jiangsu Province(BK2011276), by the Natural Science Foundation for Colleges and Universities in Jiangsu Province (11KJB11011).

(C)2014 Korean Mathematical Society 
[2]). Dual to this results, Sharp has shown for the Artinian module $A$ in [9] that the sequences $\operatorname{Att}_{R}\left(0::_{A} I^{n}\right)$ and $\operatorname{Att}_{R}\left(0:{ }_{A} I^{n}\right) /\left(0:_{A} I^{n+1}\right)$ do not depend on $n$ for $n>>0$. Next, Melkersson and Schenzel [8] showed that, for each integer $i$, the sets of prime ideals $\operatorname{Ass}_{R} \operatorname{Tor}_{i}^{R}\left(R / I^{n}, M\right)$ and $\operatorname{Att}_{R} \operatorname{Ext}_{R}^{i}\left(R / I^{n}, A\right)$ become independent of $n$ for $n>>0$. They also asked whether the set of prime ideals $\bigcup_{n>0} \operatorname{Ass}_{R} \operatorname{Ext}_{R}^{i}\left(R / I^{n}, M\right)$ is finite.

In 2001, Khashyarmanesh and Salarian [7] proved that $\operatorname{Ass}_{R} \operatorname{Ext}_{R}^{1}\left(R / I^{n}, M\right)$ is independent of $n$ for $n>>0$. Afterwards, in [5], it was proved, for an integer $t$, that if $\operatorname{Supp}_{R} H_{I}^{i}(M)$ is finite for all $i<t$, then

$$
\bigcup_{n>0}\left(\operatorname{Ass}_{R} \operatorname{Ext}_{R}^{t}\left(R / I^{n}, M\right)\right)_{>1}
$$

is a finite set. Next, by using the notion of M-sequences in dimension $>k$, Brodmann and Nhan [3] proved that, for an integer $t>0$, if $\operatorname{dim}_{R} H_{I}^{i}(M) \leq k$ for $\forall i<t$, then for $\forall j \leq t, \bigcup_{n>0}\left(\operatorname{Ass}_{R} \operatorname{Ext}_{R}^{j}\left(R / I^{n}, M\right)\right)_{\geq k}$ is contained in the finite set $\bigcup_{i=0}^{j} \operatorname{Ass}_{R} \operatorname{Ext}_{R}^{i}(R / I, M)$. Moreover, in 2008, Khashyarmanesh and Khosh-Ahang [6] proved that, for an integer $t>0$, if $\operatorname{dim}_{R} H_{I}^{i}(M) \leq k$ for $\forall i<t$, then for $\forall i \leq t$,

$$
\bigcup_{n>0}\left(\operatorname{Ass}_{R} \operatorname{Ext}_{R}^{i}\left(R / I^{n}, M\right)\right)_{\geq k} \text { and } \bigcup_{n>0}\left(\operatorname{Supp}_{R} \operatorname{Ext}_{R}^{i-1}\left(R / I^{n}, M\right)\right)_{\geq k}
$$

are two finite sets.

In this paper, by computing the local cohomology modules and Ext-modules via the injective resolution of $M$, we proved that, for an integer $t>0$, if $\operatorname{dim}_{R} H_{I}^{i}(M) \leq k$ for $\forall i<t$, then

$$
\bigcup_{i=0}^{j}\left(\operatorname{Ass}_{R} H_{I}^{i}(M)\right)_{\geq k}=\bigcup_{i=0}^{j}\left(\operatorname{Ass}_{R} \operatorname{Ext}_{R}^{i}\left(R / I^{n}, M\right)\right)_{\geq k}
$$

for $\forall j \leq t$ and $\forall n>0$. This shows that, for $\forall i \leq t$,

$$
\bigcup_{n>0}\left(\operatorname{Ass}_{R} \operatorname{Ext}_{R}^{i}\left(R / I^{n}, M\right)\right)_{\geq k} \text { and } \bigcup_{i=0}^{t}\left(\operatorname{Ass}_{R} H_{I}^{i}(M)\right)_{\geq k}
$$

are both contained in

$$
\bigcup_{i=0}^{t} \operatorname{Ass}_{R} \operatorname{Ext}_{R}^{i}(R / I, M)
$$

Also, by investigating the relationship among $M$-sequences in dimension $>k$, filter regular sequence and regular sequence, we prove that

$$
\bigcup_{i=1}^{r}\left(\operatorname{Ass}_{R} M /\left(x_{1}^{n_{1}}, x_{2}^{n_{2}}, \ldots, x_{i}^{n_{i}}\right) M\right)_{\geq k}=\bigcup_{i=1}^{r}\left(\operatorname{Ass}_{R} M /\left(x_{1}, x_{2}, \ldots, x_{i}\right) M\right)_{\geq k}
$$


if $x_{1}, x_{2}, \ldots, x_{r}$ is $M$-sequences in dimension $>k$ and $n_{1}, n_{2}, \ldots, n_{r}$ are some positive integers. This shows that

$$
\left(\operatorname{Ass}_{R} M /\left(x_{1}^{n_{1}}, x_{2}^{n_{2}}, \ldots, x_{r}^{n_{r}}\right) M\right)_{\geq k} \backslash \bigcup_{i=1}^{r-1}\left(\operatorname{Ass}_{R} M /\left(x_{1}^{n_{1}}, x_{2}^{n_{2}}, \ldots, x_{i}^{n_{i}}\right) M\right)_{\geq k}
$$

is independent of $n_{1}, n_{2}, \ldots, n_{r}$ for $n_{1}, n_{2}, \ldots, n_{r}$ large.

\section{Auxiliary and preliminary results}

Let $N$ be a non-zero $R$-module. The Krull dimension $\operatorname{dim}_{R} N$ of $N$ is the supremum of lengths of chains of prime ideals in $\operatorname{Supp}_{R} N$ if this supremum exists, and $\infty$ otherwise. In the case when $N$ is finitely generated, this is equal to $\operatorname{dim}_{R} R /(0: N)$. If $R$-module $N=0$, we set $\operatorname{dim}_{R} N=-1$.

Lemma 2.1. Assume that $0 \longrightarrow N_{1} \longrightarrow N_{2} \longrightarrow N_{3} \longrightarrow 0$ is an exact sequence of R-modules. Then $\operatorname{dim}_{R} N_{2}=\operatorname{Max}\left\{\operatorname{dim}_{R} N_{1}, \operatorname{dim}_{R} N_{3}\right\}$.

Proof. By virtue of the exactness of localization, it clear that

$$
\operatorname{Supp}_{R} N_{2}=\operatorname{Supp}_{R} N_{1} \cup \operatorname{Supp}_{R} N_{3} .
$$

Then it follows that $\operatorname{dim}_{R} N_{2}=\operatorname{Max}\left\{\operatorname{dim}_{R} N_{1}, \operatorname{dim}_{R} N_{3}\right\}$.

Lemma 2.2. Let $N$ be an R-module. Then

$$
\operatorname{Ass}_{R} \Gamma_{I}(N)=\operatorname{Ass}_{R} \operatorname{Hom}_{R}(R / I, N)=\operatorname{Ass}_{R} N \cap V(I) .
$$

Proof. It follows from [1] that $\operatorname{Ass}_{R} \operatorname{Hom}_{R}(R / I, N)=\operatorname{Ass}_{R} N \cap V(I)$. Now we will prove that

$$
\operatorname{Ass}_{R} \Gamma_{I}(N)=\operatorname{Ass}_{R} N \cap V(I) .
$$

Let $\mathfrak{p} \in \operatorname{Ass}_{R} \Gamma_{I}(N)$. Then $\Gamma_{I R_{\mathfrak{p}}}\left(N_{\mathfrak{p}}\right) \neq 0$, and then $\mathfrak{p} \in V(I)$. It is clear that $\mathfrak{p} \in \operatorname{Ass}_{R} N$. So $\operatorname{Ass}_{R} \Gamma_{I}(N) \subseteq \operatorname{Ass}_{R} N \cap V(I)$. On the other hand, let $\mathfrak{p} \in \operatorname{Ass}_{R} N \cap V(I)$. Then there exists $x \in N$ such that $\mathfrak{p}=\left(0:_{R} x\right)$. As $I \subseteq \mathfrak{p}$ we have $I x=0$, thus $x \in \Gamma_{I}(N)$. It follows that $\mathfrak{p} \in \operatorname{Ass}_{R} \Gamma_{I}(N)$. Hence, $\operatorname{Ass}_{R} \Gamma_{I}(N)=\operatorname{Ass}_{R} N \cap V(I)$. This completes the proof.

The following lemma is a well-known result. We can't find a reference for it. For the convenience of the reader, we give a proof of it.

Lemma 2.3. Let $K, L$ be two $R$-modules. If $K \subseteq L$ is an essential extension, then $\operatorname{Ass}_{R} K=\operatorname{Ass}_{R} L$.

Proof. It is clear that $\operatorname{Ass}_{R} K \subseteq \operatorname{Ass}_{R} L$. On the other hand, let $\mathfrak{p} \in \operatorname{Ass}_{R} L$. Then, there exists $x \in L, \mathfrak{p}=\operatorname{Ann}_{R} x$. Since $K \subseteq L$ is an essential extension, there exists $r \in R, r x \in K$ and $r x \neq 0$. Thus, $r \notin \mathfrak{p}$. By virtue of this, it is easy to verify that $\operatorname{Ann}_{R}(r x) \subseteq \mathfrak{p}$. This show that $\operatorname{Ann}_{R}(r x)=\operatorname{Ann}_{R} x=\mathfrak{p}$, and $\mathfrak{p} \in \operatorname{Ass}_{R} K$. Hence, $\operatorname{Ass}_{R} K=\operatorname{Ass}_{R} L$. 
Let $k \geq 0$ be an integer. Let $x_{1}, x_{2}, \ldots, x_{r}$ be a sequence of elements of $R$. We say that $x_{1}, x_{2}, \ldots, x_{r}$ is $M$-sequences in dimension $>k$ if $x_{i} \notin \mathfrak{p}$ for all $\mathfrak{p} \in\left(\operatorname{Ass}_{R} M /\left(x_{1}, x_{2}, \ldots, x_{i-1}\right) M\right)_{>k}$ and all $i=1,2, \ldots, r$ (see [3, Definition 2.1]). It is easy to see that if $x_{1}, x_{2}, \ldots, x_{r}$ is $M$-sequences in dimension $>k$, then so is $x_{1}^{n_{1}}, x_{2}^{n_{2}}, \ldots, x_{r}^{n_{r}}$ for all positive integers $n_{1}, n_{2}, \ldots, n_{r}$. For the notion of $M$-sequences in dimension $>k$, Brodmann and Nhan gave the following characterization:

Remark 2.4 ([3, Lemma 2.4]). Let $t>0$ be an integer. Then,

(i) $\operatorname{dim}_{R} H_{I}^{i}(M) \leq k$ for $\forall i<t$ if and only if there exists an $M$-sequence in dimension $>k$ of length $t$ in $I$.

(ii) If $\operatorname{dim} M / I M>k$. Then each $M$-sequence in dimension $>k$ in $I$ may be extended to a maximal $M$-sequence in dimension $>k$ in $I$. Moreover, all maximal $M$-sequences in dimension $>k$ in $I$ have the same length, this common length is equal to the least integer $i$ such that $\operatorname{dim}_{R} H_{I}^{i}(M)>k$. We usually denote this length by $\operatorname{depth}_{k}(I, M)$.

(iii) If $\operatorname{dim} M / I M \leq k$. Then there exists an $M$-sequence in dimension $>k$ in $I$ of length $n$ for any integer $n>0$.

The two lemmas below establish the relationships among a $M$-sequence in dimension $>k$, a filter regular sequence on the localization of $M$ and a regular sequence on the localization of $M$.

Lemma 2.5. Let $M$ be a finitely generated $R$-module. Let $x_{1}, x_{2}, \ldots, x_{r}$ be $M$ sequences in dimension $>k$. Then, for $\mathfrak{p} \in$ Spec $R$ satisfying $x_{1}, x_{2}, \ldots, x_{r} \subseteq \mathfrak{p}$ and $\operatorname{dim} R / \mathfrak{p} \geq k, x_{1} / 1, x_{2} / 1, \ldots, x_{r} / 1 \in \mathfrak{p} R_{\mathfrak{p}}$ is a filter regular sequence on $M_{\mathfrak{p}}$.

Proof. Let $\mathfrak{p} \in \operatorname{Spec} R$ satisfying $x_{1}, x_{2}, \ldots, x_{r} \subseteq \mathfrak{p}$ and $\operatorname{dim} R / \mathfrak{p} \geq k$. Let $\mathfrak{q} R_{\mathfrak{p}} \in$ $\operatorname{Spec} R_{\mathfrak{p}} \backslash\left\{\mathfrak{p} R_{\mathfrak{p}}\right\}$. Then $\operatorname{dim} R / \mathfrak{q}>k$. Then $x_{1} / 1, x_{2} / 1, \ldots, x_{r} / 1$ is a poor $M_{\mathfrak{q}}$ regular sequence. Note that $M_{\mathfrak{q}} \cong\left(M_{\mathfrak{p}}\right)_{\mathfrak{q} R_{\mathfrak{p}}}$. So $x_{1} / 1, x_{2} / 1, \ldots, x_{r} / 1 \in \mathfrak{p} R_{\mathfrak{p}}$ is a filter regular sequence on $M_{\mathfrak{p}}$.

For an $R$-module $K$ and an ideal $I$, we use $0:_{K}\langle I\rangle$ to denote the submodule $\left\{x \in K \mid I^{n} x=0\right.$ for some $\left.n>0\right\}$.

Lemma 2.6. Let $M$ be a finitely generated $R$-module. Let $x_{1}, x_{2}, \ldots, x_{r}$ be $M$-sequences in dimension $>k$. For $\mathfrak{p} \in$ SpecR satisfying $x_{1}, x_{2}, \ldots, x_{r} \subseteq \mathfrak{p}$ and $\operatorname{dim} R / \mathfrak{p} \geq k$, if $\mathfrak{p} \notin \operatorname{Ass}_{R} M /\left(x_{1}, x_{2}, \ldots, x_{i-1}\right) M$ for every $i, 1 \leq i \leq r$, then $x_{1} / 1, x_{2} / 1, \ldots, x_{r} / 1 \in \mathfrak{p} R_{\mathfrak{p}}$ is a poor $M_{\mathfrak{p}}$-regular sequence.

Proof. By Lemma 2.5, For $\mathfrak{p} \in \operatorname{Spec} R$ satisfying $x_{1}, x_{2}, \ldots, x_{r} \subseteq \mathfrak{p}$ and $\operatorname{dim} R / \mathfrak{p}$ $\geq k, x_{1} / 1, x_{2} / 1, \ldots, x_{r} / 1 \in \mathfrak{p} R_{\mathfrak{p}}$ is a filter regular sequence on $M_{\mathfrak{p}}$. Then by the definition of the filter regular sequence, we have that

$$
0:_{M_{\mathfrak{p}} /\left(x_{1}, x_{2}, \ldots, x_{i-1}\right) M_{\mathfrak{p}}} x_{i} \subseteq 0:_{M_{\mathfrak{p}} /\left(x_{1}, x_{2}, \ldots, x_{i-1}\right) M_{\mathfrak{p}}}\left\langle\mathfrak{p} R_{\mathfrak{p}}\right\rangle
$$


for all $i=1, \ldots, r$. Since $\mathfrak{p} R_{\mathfrak{p}} \notin \operatorname{Ass}_{R_{\mathfrak{p}}} M_{\mathfrak{p}} /\left(x_{1}, x_{2}, \ldots, x_{i-1}\right) M_{\mathfrak{p}}$ for every $i, 1 \leq$ $i \leq r, 0:_{M_{\mathfrak{p}} /\left(x_{1}, x_{2}, \ldots, x_{i-1}\right) M_{\mathfrak{p}}}\left\langle\mathfrak{p} R_{\mathfrak{p}}\right\rangle=0$ for all $i=1, \ldots, r$. So $x_{1} / 1, x_{2} / 1, \ldots$, $x_{t} / 1 \in \mathfrak{p} R_{\mathfrak{p}}$ is a poor $M_{\mathfrak{p}}$-regular sequence.

\section{Main results}

Proposition 3.1. Let $M$ be a finitely generated $R$-module and $E^{\bullet}(M)$ a minimal injective resolution of $M$. Let $k, t$ be two integers. The following are equivalent:

(i) $\operatorname{dim}_{R} H_{I}^{i}(M) \leq k$ for $\forall i<t$;

(ii) $\operatorname{dim}_{R} \operatorname{Ext}_{R}^{i}(R / I, M) \leq k$ for $\forall i<t$;

(iii) $\operatorname{dim}_{R} \Gamma_{I}\left(E^{i}(M)\right) \leq k$ for $\forall i<t$;

(iv) $\operatorname{dim}_{R} \operatorname{Hom}_{R}\left(R / I, E^{i}(M)\right) \leq k$ for $\forall i<t$.

Proof. (i) $\Longleftrightarrow$ (iii): We denote $H_{I}^{i}(\bullet)$ by $T^{i}(\bullet), i \geq 0$. and denote $\Gamma_{I}(\bullet)$ by $T(\bullet)\left(=T^{0}(\bullet)\right)$.

We have the following commutative graph:

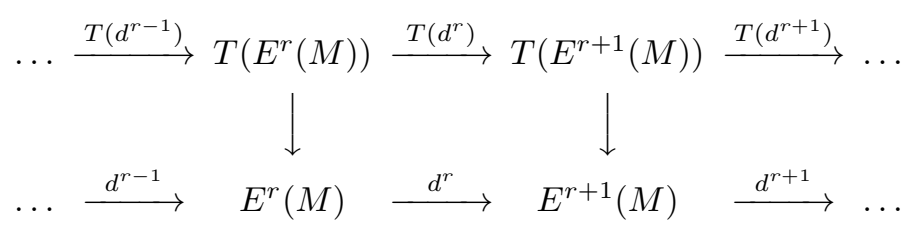

Since $\operatorname{Ker} d^{r} \subseteq E^{r}(M)$ is an essential extension, then $\operatorname{Ker} T\left(d^{r}\right)=\operatorname{Ker} d^{r} \cap$ $T\left(E^{r}(M)\right) \subseteq T\left(E^{r}(M)\right)$ is an essential extension. By Lemma 2.3,

$$
\operatorname{Ass}_{R} \operatorname{Ker} T\left(d^{r}\right)=\operatorname{Ass}_{R} T\left(E^{r}(M)\right) .
$$

Then

$$
\operatorname{Supp}_{R} \operatorname{Ker} T\left(d^{r}\right)=\operatorname{Supp}_{R} T\left(E^{r}(M)\right) .
$$

and so that

$$
\operatorname{dim}_{R} \operatorname{Ker} T\left(d^{r}\right) \leq k \text { if and only if } \operatorname{dim}_{R} T\left(E^{r}(M)\right) \leq k
$$

for some integer $k$. On the other hand, it follows by Lemma 2.1 that

$$
\operatorname{dim}_{R} \operatorname{Im} T\left(d^{r}\right) \leq k \text { if } \operatorname{dim}_{R} T\left(E^{r}(M)\right) \leq k .
$$

By using the following exact sequence

$$
0 \longrightarrow \operatorname{Im} T\left(d^{r-1}\right) \longrightarrow \operatorname{Ker} T\left(d^{r}\right) \longrightarrow T^{r}(M) \longrightarrow 0
$$

for $r=1,2, \ldots, t-1$ and $\operatorname{Ker} T\left(d^{0}\right) \cong T^{0}(M)$, it follows from the results $(a)$ and $(b)$ that

$\operatorname{dim}_{R} T^{i}(M) \leq k$ for $\forall i<t$ if and only if $\operatorname{dim}_{R} T\left(E^{i}(M)\right) \leq k$ for $\forall i<t$.

This completes the proof of (i) $\Longleftrightarrow$ (iii).

(ii) $\Longleftrightarrow$ (iv): By the same argument as above (we only replace $H_{I}^{i}(\bullet), \Gamma_{I}(\bullet)$ by $\operatorname{Ext}_{R}^{i}(R / I, \bullet), \operatorname{Hom}_{R}(R / I, \bullet)$ respectively), it follows that (ii) 

then

(iii) $\Longleftrightarrow$ (iv): By Lemma 2.2, $\operatorname{Ass}_{R} \Gamma_{I}\left(E^{i}(M)\right)=\operatorname{Ass}_{R} \operatorname{Hom}_{R}\left(R / I, E^{i}(M)\right)$,

$$
\operatorname{Supp}_{R} \Gamma_{I}\left(E^{i}(M)\right)=\operatorname{Supp}_{R} \operatorname{Hom}_{R}\left(R / I, E^{i}(M)\right),
$$

and so we have that (iii) and (iv) are equivalent.

This completes the proof.

Lemma 3.2. Let $M$ be a finitely generated $R$-module and $E^{\bullet}(M)$ a minimal injective resolution of $M$. For an integer $t>0$, if $\operatorname{dim}_{R} H_{I}^{i}(M) \leq k$ for $\forall i<t$. Then there are some equalities:

(i) $\bigcup_{i=0}^{j}\left(\operatorname{Supp}_{R} H_{I}^{i}(M)\right)_{>k}=\bigcup_{i=0}^{j}\left(\operatorname{Supp}_{R} \operatorname{Ext}_{R}^{i}(R / I, M)\right)_{>k}=\emptyset$ for $\forall j<t$.

(ii)

$$
\begin{aligned}
\bigcup_{i=0}^{j}\left(\operatorname{Ass}_{R} H_{I}^{i}(M)\right)_{k} & =\bigcup_{i=0}^{j}\left(\operatorname{Ass}_{R} \Gamma_{I}\left(E^{i}(M)\right)\right)_{k} \\
& =\bigcup_{i=0}^{j}\left(\operatorname{Ass}_{R} \operatorname{Hom}\left(R / I, E^{i}(M)\right)\right)_{k} \\
& =\bigcup_{i=0}^{j}\left(\operatorname{Ass}_{R} \operatorname{Ext}_{R}^{i}(R / I, M)\right)_{k}
\end{aligned}
$$

for $\forall j \leq t$.

(iii)

$$
\begin{aligned}
\left(\operatorname{Ass}_{R} H_{I}^{t}(M)\right)_{>k} & =\left(\operatorname{Ass}_{R} \Gamma_{I}\left(E^{t}(M)\right)\right)_{>k} \\
& =\left(\operatorname{Ass}_{R} \operatorname{Hom}\left(R / I, E^{t}(M)\right)\right)_{>k} \\
& =\left(\operatorname{Ass}_{R} \operatorname{Ext}_{R}^{t}(R / I, M)\right)_{>k} .
\end{aligned}
$$

Proof. (i) Since $\operatorname{dim}_{R} H_{I}^{i}(M) \leq k$ for $\forall i<t$, it follows by Proposition 3.1 that

$$
\bigcup_{i=0}^{j}\left(\operatorname{Supp}_{R} H_{I}^{i}(M)\right)_{>k}=\bigcup_{i=0}^{j}\left(\operatorname{Supp}_{R} \operatorname{Ext}_{R}^{i}(R / I,(M))\right)_{>k}=\varnothing
$$

for $\forall j<t$.

(ii) We denote $H_{I}^{i}(\bullet)$ by $T^{i}(\bullet), i \geq 0$. And we denote $\Gamma_{I}(\bullet)$ by $T(\bullet)(=$ $\left.T^{0}(\bullet)\right)$.

As the proof of Proposition 3.1, we have the following commutative graph:

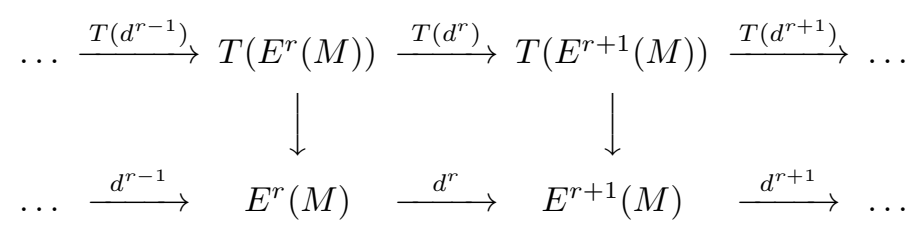

Since $\operatorname{Ker} d^{i} \subseteq E^{i}(M)$ is an essential extension, then $\operatorname{Ker} T\left(d^{i}\right)=\operatorname{Ker} d^{i} \cap$ $T\left(E^{i}(M)\right) \subseteq T\left(E^{i}(M)\right)$ is an essential extension. Then, by Lemma 2.3, for $\forall i \geq 0$,

$$
\operatorname{Ass}_{R} \operatorname{Ker} T\left(d^{i}\right)=\operatorname{Ass}_{R} T\left(E^{i}(M)\right) .
$$


Let $j \leq t$ be an integer. In the following, we use induction on $j$ to prove that

$$
\bigcup_{i=0}^{j}\left(\operatorname{Ass}_{R} T\left(E^{i}(M)\right)\right)_{k}=\bigcup_{i=0}^{j}\left(\operatorname{Ass}_{R} T^{i}(M)\right)_{k} .
$$

Let $j=0$. Since $\operatorname{Ker} T\left(d^{0}\right) \cong T^{0}(M)$, it follows from the equality (1) that

$$
\left(\operatorname{Ass}_{R} T\left(E^{0}(M)\right)\right)_{k}=\left(\operatorname{Ass}_{R} T^{0}(M)\right)_{k} .
$$

Then we suppose that $j>1$ and that the result have been proved for $j-1$ :

$$
\bigcup_{i=0}^{j-1}\left(\operatorname{Ass}_{R} T\left(E^{i}(M)\right)\right)_{k}=\bigcup_{i=0}^{j-1}\left(\operatorname{Ass}_{R} T^{i}(M)\right)_{k} .
$$

For every $i \in\{1,2, \ldots, j\}$, from the exact sequence

$$
0 \longrightarrow \operatorname{Im} T\left(d^{i-1}\right) \longrightarrow \operatorname{Ker} T\left(d^{i}\right) \longrightarrow T^{i}(M) \longrightarrow 0,
$$

it follows that

$$
\begin{aligned}
\left(\operatorname{Ass}_{R} T\left(E^{i}(M)\right)\right)_{k} & =\left(\operatorname{Ass}_{R} \operatorname{Ker} T\left(d^{i}\right)\right)_{k} \\
& \subseteq\left(\operatorname{Ass}_{R} \operatorname{Im} T\left(d^{i-1}\right)\right)_{k} \bigcup\left(\operatorname{Ass}_{R} T^{i}(M)\right)_{k} \\
& =\left(\operatorname{Supp}_{R} \operatorname{Im} T\left(d^{i-1}\right)\right)_{k} \bigcup\left(\operatorname{Ass}_{R} T^{i}(M)\right)_{k} \\
& \subseteq\left(\operatorname{Supp}_{R} T\left(E^{i-1}(M)\right)\right)_{k} \bigcup\left(\operatorname{Ass}_{R} T^{i}(M)\right)_{k} \\
& =\left(\operatorname{Ass}_{R} T\left(E^{i-1}(M)\right)\right)_{k} \bigcup\left(\operatorname{Ass}_{R} T^{i}(M)\right)_{k}
\end{aligned}
$$

Then we have that

$$
\bigcup_{i=0}^{j}\left(\operatorname{Ass}_{R} T\left(E^{i}(M)\right)\right)_{k} \subseteq \bigcup_{i=0}^{j}\left(\operatorname{Ass}_{R} T^{i}(M)\right)_{k} .
$$

On the other hand, let $\mathfrak{p} \in \bigcup_{i=0}^{j}\left(\operatorname{Ass}_{R} T^{i}(M)\right)_{k}$. If $\mathfrak{p} \in \bigcup_{i=0}^{j-1}\left(\operatorname{Ass}_{R} T^{i}(M)\right)_{k}$, by the inductive assumption, it is clear that $\mathfrak{p} \in \bigcup_{i=0}^{j}\left(\operatorname{Ass}_{R} T\left(E^{i}(M)\right)\right)_{k}$. So we assume that $\mathfrak{p} \notin \bigcup_{i=0}^{j-1}\left(\operatorname{Ass}_{R} T^{i}(M)\right)_{k}$. Then, by the inductive assumption again,

$$
\mathfrak{p} \notin \bigcup_{i=0}^{j-1}\left(\operatorname{Ass}_{R} T\left(E^{i}(M)\right)\right)_{k}=\bigcup_{i=0}^{j-1}\left(\operatorname{Supp}_{R} T\left(E^{i}(M)\right)\right)_{k}
$$

and $\mathfrak{p} \notin \operatorname{Supp}_{R} \operatorname{Im} T\left(d^{j-1}\right)$. The exact sequence

$$
0 \longrightarrow \operatorname{Im} T\left(d^{j-1}\right) \longrightarrow \operatorname{Ker} T\left(d^{j}\right) \longrightarrow T^{j}(M) \longrightarrow 0
$$

implies that

$$
\left(\operatorname{Ker} T\left(d^{j}\right)\right)_{\mathfrak{p}} \cong\left(T^{j}(M)\right)_{\mathfrak{p}}
$$

Then, since $\mathfrak{p} \in\left(\operatorname{Ass}_{R} T^{j}(M)\right)_{k}$, it follows that

$$
\mathfrak{p} R_{\mathfrak{p}} \in \operatorname{Ass}_{R_{\mathfrak{p}}} T^{j}(M)_{\mathfrak{p}}=\operatorname{Ass}_{R_{\mathfrak{p}}} \operatorname{Ker} T\left(d^{j}\right)_{\mathfrak{p}}
$$


and so by the equality (1), $\mathfrak{p} \in\left(\operatorname{Ass}_{R} \operatorname{Ker} T\left(d^{j}\right)\right)_{k}=\left(\operatorname{Ass}_{R} T\left(E^{j}(M)\right)_{k}\right.$. Hence, $\mathfrak{p} \in \bigcup_{i=0}^{j}\left(\operatorname{Ass}_{R} T\left(E^{i}(M)\right)\right)_{k}$, and

$$
\bigcup_{i=0}^{j}\left(\operatorname{Ass}_{R} T^{i}(M)\right)_{k} \subseteq \bigcup_{i=0}^{j}\left(\operatorname{Ass}_{R} T\left(E^{i}(M)\right)\right)_{k} .
$$

This shows the equality in the previous formula.

Thus, we have that

$$
\bigcup_{i=0}^{j}\left(\operatorname{Ass}_{R} H_{I}^{i}(M)\right)_{k}=\bigcup_{i=0}^{j}\left(\operatorname{Ass}_{R} \Gamma_{I}\left(E^{i}(M)\right)\right)_{k}
$$

for $\forall j \leq t$.

By the same argument as above (we only replace $H_{I}^{i}(\bullet), \Gamma_{I}(\bullet)$ by $\operatorname{Ext}_{R}^{i}(R / I$,

$\bullet$ ), $\operatorname{Hom}_{R}(R / I, \bullet)$, respectively), we have that

$$
\bigcup_{i=0}^{j}\left(\operatorname{Ass}_{R} \operatorname{Ext}_{R}^{i}(R / I, M)\right)_{k}=\bigcup_{i=0}^{j}\left(\operatorname{Ass}_{R} \operatorname{Hom}\left(R / I, E^{i}(M)\right)\right)_{k}
$$

for $\forall j \leq t$.

Finally, the result (ii) follows from Lemma 2.2.

(iii) We continue to use the notations as (ii). Since $\operatorname{dim}_{R} H_{I}^{i}(M) \leq k$ for $\forall i<t$, it follows that $\operatorname{dim}_{R} \operatorname{Im} T\left(d^{t-1}\right) \leq k$ by $((\mathrm{i}) \Longleftrightarrow$ (iii)) of Proposition 3.1. From the exact sequences

$$
0 \longrightarrow \operatorname{Im} T\left(d^{t-1}\right) \longrightarrow \operatorname{Ker} T\left(d^{t}\right) \longrightarrow T^{t}(M) \longrightarrow 0,
$$

it follows that for $\forall \mathfrak{p} \in \operatorname{Spec} R$ satisfying $\operatorname{dim} R / \mathfrak{p}>k$, we have that

$$
\left(\operatorname{Ker} T\left(d^{t}\right)\right)_{\mathfrak{p}} \cong\left(T^{t}(M)\right)_{\mathfrak{p}}
$$

Then, $\left.\mathfrak{p} \in \operatorname{Ass}_{R} \operatorname{Ker} T\left(d^{t}\right)\right)_{>k}$ if and only if $\mathfrak{p} \in\left(\operatorname{Ass}_{R} T^{t}(M)\right)_{>k}$. Hence, by the equality (1)

$$
\left.\left(\operatorname{Ass}_{R} T^{t}(M)\right)_{>k}=\operatorname{Ass}_{R} \operatorname{Ker} T\left(d^{t}\right)\right)_{>k}=\left(\operatorname{Ass}_{R} T\left(E^{t}(M)\right)\right)_{>k} .
$$

This shows that

$$
\left(\operatorname{Ass}_{R} H_{I}^{t}(M)\right)_{>k}=\left(\operatorname{Ass}_{R} \Gamma_{I}\left(E^{t}(M)\right)\right)_{>k} .
$$

By the same argument as above, it follows that

$$
\left(\operatorname{Ass}_{R} \operatorname{Hom}\left(R / I, E^{t}(M)\right)_{>k}=\left(\operatorname{Ass}_{R} \operatorname{Ext}_{R}^{t}(R / I, M)\right)_{>k} .\right.
$$

Then by Lemma 2.2 , the result (iii) follows.

Note that $H_{I}^{i}(M) \cong H_{I^{n}}^{i}(M)$ for any positive integer $n$. The following corollaries are two immediate consequences of Theorem 3.2. 
Corollary 3.3. Let $M$ be a finitely generated $R$-module. For an integer $t>0$, $\operatorname{dim}_{R} H_{I}^{i}(M) \leq k$ for $\forall i<t$. Then

$$
\bigcup_{i=0}^{j}\left(\operatorname{Ass}_{R} H_{I}^{i}(M)\right)_{\geq k}=\bigcup_{i=0}^{j}\left(\operatorname{Ass}_{R} \operatorname{Ext}_{R}^{i}\left(R / I^{n}, M\right)\right)_{\geq k}
$$

for $\forall j \leq t$ and $\forall n>0$. In particular, $\left(\operatorname{Ass}_{R} H_{I}^{i}(M)\right)_{\geq k}$ is a finite set for $\forall i \leq t$.

Corollary 3.4 ([6, Theorem 1.1]). Let $M$ be a finitely generated R-module. For an integer $t>0, \operatorname{dim}_{R} H_{I}^{i}(M) \leq k$ for $\forall i<t$. Then

(i) $\bigcup_{n>0}\left(\operatorname{Ass}_{R} \operatorname{Ext}_{R}^{i}\left(R / I^{n}, M\right)\right)_{\geq k} \subseteq \bigcup_{i=0}^{t} \operatorname{Ass}_{R} \operatorname{Ext}_{R}^{i}(R / I, M)$ for $\forall i \leq t$. In particular, $\bigcup_{n>0}\left(\operatorname{Ass}_{R} \operatorname{Ext}_{R}^{i}\left(R / I^{n}, M\right)\right)_{\geq k}$ is a finite set for $\forall i \leq t$.

(ii) $\bigcup_{n>0}\left(\operatorname{Supp}_{R} \operatorname{Ext}_{R}^{i}\left(R / I^{n}, M\right)\right)_{\geq k}=\bigcup_{n>0}\left(\operatorname{Ass}_{R} \operatorname{Ext}_{R}^{i}\left(R / I^{n}, M\right)\right)_{\geq k}$ for $\forall i<t$. In particular, $\bigcup_{n>0}\left(\operatorname{Supp}_{R} \operatorname{Ext}_{R}^{i}\left(R / I^{n}, M\right)\right)_{\geq k}$ is a finite set for $\forall i<t$.

Theorem 3.5. Let $M$ be a finitely generated $R$-module. Let $x_{1}, x_{2}, \ldots, x_{r}$ be $M$-sequences in dimension $>k$. Then, for any positive integers $n_{1}, n_{2}, \ldots, n_{r}$,

(i) $\left(\operatorname{Ass}_{R} M /\left(x_{1}^{n_{1}}, x_{2}^{n_{2}}, \ldots, x_{i}^{n_{i}}\right) M\right)_{>k} \cap V\left(x_{i+1}\right)=\emptyset, \forall i<r$;

(ii) $\left(\operatorname{Ass}_{R} M /\left(x_{1}^{n_{1}}, x_{2}^{n_{2}}, \ldots, x_{r}^{n_{r}}\right) M\right)_{>k}=\left(\operatorname{Ass}_{R} M /\left(x_{1}, x_{2}, \ldots, x_{r}\right) M\right)_{>k}$;

(iii) $\bigcup_{i=1}^{r}\left(\operatorname{Ass}_{R} M /\left(x_{1}^{n_{1}}, x_{2}^{n_{2}}, \ldots, x_{i}^{n_{i}}\right) M\right)_{k}=\bigcup_{i=1}^{r}\left(\operatorname{Ass}_{R} M /\left(x_{1}, x_{2}, \ldots, x_{i}\right) M\right)_{k}$. In particular,

$$
\bigcup_{n_{1}, n_{2}, \ldots, n_{r}}\left(\operatorname{Ass}_{R} M /\left(x_{1}^{n_{1}}, x_{2}^{n_{2}}, \ldots, x_{r}^{n_{r}}\right) M\right)_{\geq k}
$$

is a finite set.

Proof. (i) Let $\forall i<r$. For $\mathfrak{p} \in \operatorname{Ass}_{R} M /\left(x_{1}^{n_{1}}, x_{2}^{n_{2}}, \ldots, x_{i}^{n_{i}}\right) M \cap V\left(x_{i+1}\right)$, then, by the definition of $M$-sequences in dimension $>k, \operatorname{dim} R / \mathfrak{p} \leq k$. So

$$
\left(\operatorname{Ass}_{R} M /\left(x_{1}^{n_{1}}, x_{2}^{n_{2}}, \ldots, x_{i}^{n_{i}}\right) M\right)_{>k} \cap V\left(x_{i+1}\right)=\emptyset .
$$

(ii) Let $n_{1}, n_{2}, \ldots, n_{r}$ be any positive integers and

$$
\mathfrak{p} \in\left(\operatorname{Ass}_{R} M /\left(x_{1}^{n_{1}}, x_{2}^{n_{2}}, \ldots, x_{r}^{n_{r}}\right) M\right)_{>k} .
$$

Then $x_{1}^{n_{1}} / 1, x_{2}^{n_{2}} / 1, \ldots, x_{r}^{n_{r}} / 1 \in \mathfrak{p} R_{\mathfrak{p}}$ is a poor $M_{\mathfrak{p}}$-regular sequence. By [4, Lemma 1.2.4],

$$
\begin{aligned}
& \operatorname{Hom}\left(R_{\mathfrak{p}} /\left(x_{1}, x_{2}, \ldots, x_{r}\right) R_{\mathfrak{p}}, M_{\mathfrak{p}} /\left(x_{1}^{n_{1}}, x_{2}^{n_{2}}, \ldots, x_{r}^{n_{r}}\right) M_{\mathfrak{p}}\right) \\
& \cong \operatorname{Ext}_{R_{\mathfrak{p}}}^{r}\left(R_{\mathfrak{p}} /\left(x_{1}, x_{2}, \ldots, x_{r}\right) R_{\mathfrak{p}}, M_{\mathfrak{p}}\right) .
\end{aligned}
$$

So we have that

$$
\mathfrak{p} R_{\mathfrak{p}} \in\left(\operatorname{Ass}_{R_{\mathfrak{p}}} M_{\mathfrak{p}} /\left(x_{1}^{n_{1}}, x_{2}^{n_{2}}, \ldots, x_{r}^{n_{r}}\right) M_{\mathfrak{p}}\right)_{>k}
$$

if and only if

$$
\mathfrak{p} R_{\mathfrak{p}} \in\left(\operatorname{Ass}_{R_{\mathfrak{p}}} \operatorname{Ext}_{R_{\mathfrak{p}}}^{r}\left(R_{\mathfrak{p}} /\left(x_{1}, x_{2}, \ldots, x_{r}\right) R_{\mathfrak{p}}, M_{\mathfrak{p}}\right)\right)_{>k} .
$$

This shows that

$$
\mathfrak{p} R_{\mathfrak{p}} \in\left(\operatorname{Ass}_{R_{\mathfrak{p}}} M_{\mathfrak{p}} /\left(x_{1}^{n_{1}}, x_{2}^{n_{2}}, \ldots, x_{r}^{n_{r}}\right) M_{\mathfrak{p}}\right)_{>k}
$$


if and only if

$$
\mathfrak{p} R_{\mathfrak{p}} \in\left(\operatorname{Ass}_{R_{\mathfrak{p}}} M_{\mathfrak{p}} /\left(x_{1}, x_{2}, \ldots, x_{r}\right) M_{\mathfrak{p}}\right)_{>k}
$$

Hence,

if and only if

$$
\mathfrak{p} \in\left(\operatorname{Ass}_{R} M /\left(x_{1}^{n_{1}}, x_{2}^{n_{2}}, \ldots, x_{r}^{n_{r}}\right) M\right)_{>k}
$$

$$
\mathfrak{p} \in\left(\operatorname{Ass}_{R} M /\left(x_{1}, x_{2}, \ldots, x_{r}\right) M\right)_{>k} .
$$

(iii) Since $x_{1}, x_{2}, \ldots, x_{r}$ is $M$-sequences in dimension $>k$, then, for all positive integers $n_{1}, n_{2}, \ldots, n_{r}$, so is $x_{1}^{n_{1}}, x_{2}^{n_{2}}, \ldots, x_{r}^{n_{r}}$. The result follows by the more general statement of Theorem 3.6 (proved in the following) for $I=$ $\left(x_{1}, x_{2}, \ldots, x_{r}\right)$.

The following theorem is a generalization of [3, Theorem 1.2].

Theorem 3.6. Let $M$ be a finitely generated $R$-module. Let $x_{1}, x_{2}, \ldots, x_{r} \in I$ be $M$-sequences in dimension $>k$. Then

$$
\left(\bigcup_{i=0}^{r} A s s_{R} E x t_{R}^{i}(R / I, M)\right)_{\geq k}=\left(\bigcup_{i=0}^{r}\left(A s s_{R} M /\left(x_{1}, x_{2}, \ldots, x_{i}\right) M\right)_{\geq k} \cap V(I) .\right.
$$

In particular,

$$
\left(\bigcup_{n} A s s_{R} E x t_{R}^{r}\left(R / I^{n}, M\right)\right)_{\geq k}
$$

is contained in the finite set

$$
\left(A s s_{R} M /\left(x_{1}, x_{2}, \ldots, x_{r}\right) M\right)_{>k} \cup\left(\bigcup_{i=0}^{r} A s s_{R} M /\left(x_{1}, x_{2}, \ldots, x_{i}\right) M\right)_{k} .
$$

Proof. We use induction on $t$ to prove that

$$
\left(\bigcup_{i=0}^{t} \operatorname{Ass}_{R} \operatorname{Ext}_{R}^{i}(R / I, M)\right)_{\geq k}=\left(\bigcup_{i=0}^{t}\left(\operatorname{Ass}_{R} M /\left(x_{1}, x_{2}, \ldots, x_{i}\right) M\right)_{\geq k} \cap V(I)\right.
$$

for every $t, 0 \leq t \leq r$. When $t=0$, then it is nothing to prove since it is well know that $\operatorname{Ass}_{R} \operatorname{Hom}(R / I, M)=\operatorname{Ass}_{R} M \cap V(I)$. Then we suppose that $t>1$ and that the result have been proved for $t-1$ :

$$
\left(\bigcup_{i=0}^{t-1} \operatorname{Ass}_{R} \operatorname{Ext}_{R}^{i}(R / I, M)\right)_{\geq k}=\left(\bigcup_{i=0}^{t-1}\left(\operatorname{Ass}_{R} M /\left(x_{1}, x_{2}, \ldots, x_{i}\right) M\right)_{\geq k} \cap V(I) .\right.
$$

Let $\mathfrak{p} \in\left(\bigcup_{i=0}^{t}\left(\operatorname{Ass}_{R} M /\left(x_{1}, x_{2}, \ldots, x_{i}\right) M\right)_{\geq k} \cap V(I)\right.$.

If $\mathfrak{p} \in \bigcup_{i=0}^{t-1} \operatorname{Ass}_{R} M /\left(x_{1}, x_{2}, \ldots, x_{i}\right) M$, then by the inductive assumption, we have that

$$
\mathfrak{p} \in\left(\bigcup_{i=0}^{t} \operatorname{Ass}_{R} \operatorname{Ext}_{R}^{i}(R / I, M)\right)_{\geq k}
$$


If $\mathfrak{p} \notin \bigcup_{i=0}^{t-1} \operatorname{Ass}_{R} M /\left(x_{1}, x_{2}, \ldots, x_{i}\right) M$. Then $\mathfrak{p} \in \operatorname{Ass}_{R} M /\left(x_{1}, x_{2}, \ldots, x_{t}\right) M$, moreover by Lemma $2.6, x_{1} / 1, x_{2} / 1, \ldots, x_{t} / 1 \in I R_{\mathfrak{p}}$ is a poor $M_{\mathfrak{p}}$-regular sequence. Then there is an isomorphism:

$$
\operatorname{Hom}\left(R_{\mathfrak{p}} / I R_{\mathfrak{p}}, M_{\mathfrak{p}} /\left(x_{1}, x_{2}, \ldots, x_{t}\right) M_{\mathfrak{p}}\right) \cong \operatorname{Ext}_{R_{\mathfrak{p}}}^{t}\left(R_{\mathfrak{p}} / I R_{\mathfrak{p}}, M_{\mathfrak{p}}\right) .
$$

Since $\mathfrak{p} \in \operatorname{Ass}_{R} M /\left(x_{1}, x_{2}, \ldots, x_{t}\right) M$, it follows that

$$
\mathfrak{p} R_{\mathfrak{p}} \in \operatorname{Ass}_{R_{\mathfrak{p}}} M_{\mathfrak{p}} /\left(x_{1}, x_{2}, \ldots, x_{t}\right) M_{\mathfrak{p}} \cap V\left(I R_{\mathfrak{p}}\right)=\operatorname{Ass}_{R \mathfrak{p}} \operatorname{Ext}_{R_{\mathfrak{p}}}^{t}\left(R_{\mathfrak{p}} / I R_{\mathfrak{p}}, M_{\mathfrak{p}}\right) .
$$

This shows that $\mathfrak{p} \in \operatorname{Ass}_{R} \operatorname{Ext}_{R}^{t}(R / I, M)$. Therefore, we have that

$$
\left(\bigcup_{i=0}^{t}\left(\operatorname{Ass}_{R} M /\left(x_{1}, x_{2}, \ldots, x_{i}\right) M\right)_{\geq k} \cap V(I) \subseteq\left(\bigcup_{i=0}^{t} \operatorname{Ass}_{R} \operatorname{Ext}_{R}^{i}(R / I, M)\right)_{\geq k} .\right.
$$

On the other hand, let $\mathfrak{p} \in\left(\bigcup_{i=0}^{t} \operatorname{Ass}_{R} \operatorname{Ext}_{R}^{i}(R / I, M)\right)_{\geq k}$.

If $\mathfrak{p} \in \bigcup_{i=0}^{t-1} \operatorname{Ass}_{R} \operatorname{Ext}_{R}^{i}(R / I, M)$, it is clear that

$$
\mathfrak{p} \in\left(\bigcup_{i=0}^{t}\left(\operatorname{Ass}_{R} M /\left(x_{1}, x_{2}, \ldots, x_{i}\right) M\right)_{\geq k} \cap V(I)\right.
$$

by the inductive assumption.

If $\mathfrak{p} \notin \bigcup_{i=0}^{t-1} \operatorname{Ass}_{R} \operatorname{Ext}_{R}^{i}(R / I, M)$, by the inductive assumption, we have that $\mathfrak{p} \notin \bigcup_{i=0}^{t-1} \operatorname{Ass}_{R} M /\left(x_{1}, x_{2}, \ldots, x_{i}\right) M$. This shows that $x_{1} / 1, x_{2} / 1, \ldots, x_{t} / 1 \in$ $I R_{\mathfrak{p}}$ is a poor $M_{\mathfrak{p}}$-regular sequence by Lemma 2.6. Then, similar to the proof above, we can also prove that

$$
\mathfrak{p} \in\left(\bigcup_{i=0}^{t}\left(\operatorname{Ass}_{R} M /\left(x_{1}, x_{2}, \ldots, x_{i}\right) M\right)_{\geq k} \cap V(I) .\right.
$$

Therefore,

$$
\left(\bigcup_{i=0}^{t} \operatorname{Ass}_{R} \operatorname{Ext}_{R}^{i}(R / I, M)\right)_{\geq k} \subseteq\left(\bigcup_{i=0}^{t}\left(\operatorname{Ass}_{R} M /\left(x_{1}, x_{2}, \ldots, x_{i}\right) M\right)_{\geq k} \cap V(I) .\right.
$$

This proves the equality in the previous formula.

By Theorem 3.5(i), $\left(\bigcup_{i=0}^{r-1} \operatorname{Ass}_{R} M /\left(x_{1}, x_{2}, \ldots, x_{i}\right) M\right)_{>k} \cap V(I)=\emptyset$. So we have that

$$
\left(\bigcup_{n} \operatorname{Ass}_{R} \operatorname{Ext}_{R}^{r}\left(R / I^{n}, M\right)\right)_{\geq k}
$$

is contained in the finite set

$$
\left(\operatorname{Ass}_{R} M /\left(x_{1}, x_{2}, \ldots, x_{r}\right) M\right)_{>k} \cup\left(\bigcup_{i=0}^{r} \operatorname{Ass}_{R} M /\left(x_{1}, x_{2}, \ldots, x_{i}\right) M\right)_{k} .
$$

Acknowledgment. The authors thank the referee for his or her carefully reading of this manuscript. 


\section{References}

[1] N. Bourbaki, Commutative Algebra, Translated from the French. Hermann, Paris, Addison-Wesley Publishing Co., Reading, Mass., 1972.

[2] M. Brodmann, Asymptotic stability of $A s s_{R} M / I^{n} M$, Proc. Amer. Math. Soc. 74 (1979), no. $1,16-18$.

[3] M. Brodmann and L. T. Nhan, A finiteness result for associated primes of certain Extmodules, Comm. Algebra 36 (2008), no. 4, 1527-1536.

[4] W. Bruns and J. Herzog, Cohen-Macaulay Rings, Cambridge University Press, Cambridge, 1998.

[5] K. Khashyarmanesh, On the finiteness properties of $\bigcup_{i}\left(A_{s s_{R}} \operatorname{Ext}_{R}^{n}\left(R / \mathfrak{a}^{i}, M\right)\right.$, Comm. Algebra 34 (2006), no. 2, 779-784

6] K. Khashyarmanesh and F. Khosh-Ahang, Asymptotic behaviour of certain sets of associated prime ideals of Ext-modules, Manuscripta Math. 125 (2008), 345-352.

[7] K. Khashyarmanesh and Sh. Salarian, Asymptotic stability of $\operatorname{Att}_{R} \operatorname{Tor}_{1}^{R}\left(R / \mathfrak{a}^{n}, A\right)$, Proc. Edinb. Math. Soc. 44 (2001), no. 3, 479-483.

[8] L. Melkersson and P. Schenzel, Asymptotic prime ideals related to derived functors, Proc. Amer. Math. Soc. 117 (1993), no. 4, 935-938.

[9] R. Y. Sharp, Asymptotic behaviour of certain sets of attached prime ideals, J. London Math. Soc. 34 (1986), no. 2, 212-218.

LiZHONG CHU

Department of Mathematics

SOOCHOW UNIVERSITY

Suzhou 215006, P. R. ChinA

E-mail address: chulizhong@suda.edu.cn

XIAN WANG

Department of Mathematics

China University of Mining and Technology

Xuzhou, 221116, P. R. China

E-mail address: wx2008117@cumt.edu.cn 